\title{
Do copper and iron influence alcohol transformations under hydrothermal conditions?
}

\author{
ZIMING YANG ${ }^{1}$, YIJU LIAO ${ }^{1}$, ALEXANDRIA ASPIN ${ }^{1}$ \\ 'Department of Chemistry, Oakland University, Rochester, \\ MI 48309, USA (zimingyang@ oakland.edu)
}

Organic reactions in hydrothermal systems are unique and are of importance to many geochemically relevant processes such as the deep ocean carbon cycle. Reaction pathways that link organic functional groups under hydrothermal conditions have been proposed and verified by laboratory-simulated hydrothermal experiments. In these hydrothermal organic functional group interconversions, alcohols serve as a critical intermediate that connects hydrocarbons and carboxylic acids. Dehydration of alcohols to form hydrocarbons such as alkenes is favorable under hydrothermal conditions, whereas oxidation of alcohols to aldehydes and carboxylic acids is usually less likely to occur due to the requirement of an oxidizing power. In this study, we examined the effects of a few Earth-abundant materials, including a suite of iron and copper salts, on hydrothermal reaction of alcohols using several model alcohol compounds. In the absence of dissolved metals, we observe that the dehydration of alcohols is the dominant pathway in the hydrothermal fluids. In the presence of ferric or cupric ions, however, the oxidation of alcohols is greatly promoted and becomes more competitive, forming aldehydes and carboxylic acids as the major products. Our results suggest a strong oxidizing role of dissolved iron and copper in hydrothermal oxidation of alcohols, which may provide some new insights into understanding how inorganic materials control organic transformations in natural hydrothermal environments. 